\title{
Radiative Entropy Production
}

\author{
Vedat S. Arpaci* \\ University of Michigan, Ann Arbor, Michigan
}

\section{$\mathbf{T}$} HE radiative tensor obtained from the specular moments of the transfer equation is considered. The radiative entropy production is expressed in terms of this tensor.

\section{Contents}

The theory of gas radiation dates back to studies Rayleigh made over a century ago on the illumination and polarization of the sunlit sky. Since then, the theory has rapidly grown because of the efforts of astrophysicists and later of applied scientists and engineers. However, the entropy production associated with radiation apparently remains untreated and is the motivation of this study.

As is well known, the entropy production results from dissipative processes (involving mass, species, momentum, and/or heat transfer and electromagnetic or nuclear transport). Less known is the fact that the dissipation may have a diffusive or hysteretic origin, the diffusion being directional and the hysteresis being cyclic. However, except for a few cases (such as strain hardening and the magnetic saturation), the majority of dissipative processes (including the dissipation of radiation) are of diffusive nature. A recent study by Arpaci ${ }^{1}$ shows, in terms of the radiative stress obtained from the specular (kinetic) moments of the transfer equation, the diffusive nature of radiation for any optical thickness. Accordingly, the expression to be developed for entropy production is in terms of this stress and includes also the dissipation resulting from the conduction of heat and viscous friction. First, some remarks on the radiative stress are needed. These will be made in terms of spectrally averaged radiation because of its simplicity. A monochromatic approach, which may be needed for a quantitative study, is not essential here because of the conceptual nature of the intended study.

As pointed out by Felske and Tien, ${ }^{2}$ there are a variety of practical situations in which scattering is not important. For these situations, consider the spectrally averaged transfer equation,

$$
l_{i} \frac{\partial I}{\partial x_{i}}=\kappa\left(I_{0}-I\right)
$$

where $I$ denotes the intensity, $I_{0}$ its equilibrium, $\kappa$ the absorption coefficient, $l_{i}$ the direction of optical path, and $x_{i}$ the Cartesian coordinate. The usual definitions of the radiative internal energy, heat flux, and stress in terms of the intensity are

$$
u^{R}=\frac{1}{c} \int_{\Omega} I \mathrm{~d} \Omega=\frac{1}{c} J
$$

\footnotetext{
Presented as Paper 85-0408 at the AIAA 23rd Aerospace Sciences Meeting, Reno, NV, Jan. 14-17, 1985; received Feb. 27, 1986; synoptic submitted Oct. 7, 1985. Copyright (c) American Institute of Aeronautics and Astronautics, Inc., 1985. All rights reserved. Full paper available from AIAA Library, $555 \mathrm{~W}$. 57th Street, New York, NY 10019. Price: microfiche, $\$ 4.00$; hard copy, $\$ 9.00$. Remittance must accompany order.
}

*Professor of Mechanical Engineering and Applied Mechanics.

$$
\begin{gathered}
q_{i}^{R}=\int_{\Omega} I_{i} \mathrm{~d} \Omega \\
\tau_{i j}^{R}=\frac{1}{c} \int_{\Omega} I l_{i} l_{j} \mathrm{~d} \Omega=\frac{1}{c} \Pi_{i j}
\end{gathered}
$$

where the $J$ scalar and the $\Pi_{i j}$ tensor are introduced for notational convenience, $c$ is the velocity of light, and $\Omega$ is the solid angle. In terms of these definitions, the first three specular moments of the transfer equation are

$$
\begin{gathered}
\frac{\partial q_{i}^{R}}{\partial x_{i}}=\kappa_{P}(B-J) \\
\frac{\partial \Pi_{i j}}{\partial x_{j}}=-\kappa_{R} q_{i}^{R} \\
\mathrm{II}_{i j}=\frac{1}{3} B \delta_{i j}-\frac{1}{\kappa_{M}} \frac{\partial}{\partial x_{k}} \int_{\Omega} I l_{i} l_{j} l_{k} \mathrm{~d} \Omega
\end{gathered}
$$

where $B=4 E_{b} ; E_{b}=\sigma T^{4}$, the Stefan-Boltzmann law for the blackbody emissive power; $\kappa_{P}$ and $\kappa_{R}$ the Planck and Rosseland means of the absorption coefficient, respectively; and $\kappa_{M}=\left(\kappa_{P} \kappa_{R}\right)^{1 / 2}$, the geometric mean of these coefficients. The incorporation of $\kappa_{P}$ and $\kappa_{R}$ into the foregoing equations is discussed in Refs. 3-11. Clearly, Eq. (5) denotes the thermal balance, Eq. (6) the momentum balance associated with radiation, and Eq. (7) the definition of the $\Pi_{i j}$ tensor. A procedure for the evaluation of this tensor in terms of the Wallis integrals is described in Unno and Spiegel. ${ }^{12}$ This procedure leads to

$$
\Pi_{i j}=\sum_{n=0}^{\infty} \frac{\nabla^{2 n-2}\left(2 n \partial_{i} \partial_{j}+\nabla^{2} \delta_{i j}\right) B}{\kappa_{M}^{2 n}(2 n+1)(2 n+3)}
$$

where $\partial_{i} \equiv \partial / \partial x_{i}$ and $\partial_{j} \equiv \partial / \partial x_{j}$ are used for notational convenience. The same result may be found also in earlier works (see, for example, Ref. 13). The formal similarity of Eq. (8) to the Hookean constitution for elastic solids should be noted.

An alternate from for this stress may be given in terms of the isotropic radiative pressure. First, invoking the assumption of isotropy, Eqs. (2) and (4) are related as

$$
\tau_{i j}^{R}=1 / 3 u^{R} \delta_{i j}
$$

or, equivalently, as

$$
\tau_{i j}^{R}=1 / 3 \Pi_{k k} \delta_{i j}=1 / 3 J \delta_{i j}
$$

where

$$
1 / 3 \Pi_{k k}=-p
$$

is the (isotropic) pressure of radiation. Then, from the trace of $\Pi_{i j}$, noting that $l_{k} l_{k}=1$,

$$
\Pi_{k k}=\sum_{n=0}^{\infty}\left(\frac{\nabla^{2}}{\kappa_{M}^{2}}\right)^{n} \frac{B}{(2 n+1)}
$$


Now, in a manner similar to the inclusion of the isotropic pressure to the development of viscous stress from elastic stress (e.g., see Ref. 14), adding the identity

$$
1 / 3 J \delta_{i j}-1 / 3 \Pi_{k k} \delta_{i j}=0
$$

to Eq. (8), the $\Pi_{i j}$ tensor may be rearranged in terms of the radiative pressure,

$$
\Pi_{i j}=\frac{1}{3} J \delta_{i j}+\sum_{n=0}^{\infty} \frac{2 n \nabla^{2 n-2}\left(\partial_{i} \partial_{j}-1 / 3 \nabla^{2} \delta_{i j}\right) B}{\kappa_{M}^{2 n}(2 n+1)(2 n+3)}
$$

The operational similarity of Eq. (14) to the viscous (Stokesean) stress and the electromagnetic (Maxwell) stress should be noted. The use of the first term of Eq. (14) in place of Eq. (8) is the well-known Eddington approximation. The rest of the study develops an expression for the radiative entropy production in terms of $\Pi_{i j}$ given by Eqs. (8) and (14).

As is well known, the major contribution of thermal radiation to thermomechanics is the radiation heat flux $q_{i}^{R}$ (e.g., see Refs. 15-19). Accordingly, with the addition of this flux, the thermal energy becomes, in terms of the usual notation,

$$
\rho \frac{\mathrm{D} u}{\mathrm{D} t}+p\left(\frac{\partial v_{i}}{\partial x_{i}}\right)=-\frac{\partial}{\partial x_{i}}\left(q_{i}^{K}+q_{i}^{R}\right)+\tau_{i j} s_{i j}
$$

and the balance of entropy becomes

$$
\rho \frac{\mathrm{D} s}{\mathrm{D} t}=-\frac{\partial}{\partial x_{i}}\left(\frac{q_{i}^{K}+q_{i}^{R}}{T}\right)+s^{\prime \prime \prime}
$$

Now, consider the Gibbs (thermodynamic) relation

$$
\rho \frac{\mathrm{D} u}{\mathrm{D} t}=\rho T \frac{\mathrm{D} s}{\mathrm{D} t}+\frac{p}{\rho} \frac{\mathrm{D} \rho}{\mathrm{D} t}
$$

and rearrange it in terms of the conservation of mass to get

$$
\rho \frac{\mathrm{D} u}{\mathrm{D} t}=\rho T \frac{\mathrm{D} s}{\mathrm{D} t}-p\left(\frac{\partial v_{i}}{\partial x_{i}}\right)
$$

Eliminating the internal energy and the entropy among Eqs. (15), (16), and (18) yields the expression for entropy production

$$
s^{\prime \prime \prime}=\frac{1}{T}\left[-\frac{1}{T}\left(q_{i}^{K}+q_{i}^{R}\right)\left(\frac{\partial T}{\partial x_{i}}\right)+\tau_{i j} s_{i j}\right]
$$

Inserting the usual conductive and viscous constitutions

$$
q_{i}^{K}=-k\left(\frac{\partial T}{\partial x_{i}}\right), \quad \tau_{i j}=2 \mu s_{i j}
$$

and the radiative constitution from Eq. (6)

$$
q_{i}^{R}=-\frac{1}{\kappa_{R}}\left(\frac{\partial \Pi_{i j}}{\partial x_{j}}\right)
$$

into this expression gives

$$
s^{\prime \prime \prime}=\frac{1}{T}\left\{\frac{1}{T}\left[k\left(\frac{\partial T}{\partial x_{i}}\right)+\frac{1}{\kappa_{R}}\left(\frac{\partial \Pi_{i j}}{\partial x_{j}}\right)\right]\left(\frac{\partial T}{\partial x_{i}}\right)+2 \mu s_{i j} s_{i j}\right\}
$$

Explicitly, in terms of Eq. (8),

$$
\begin{aligned}
s^{\prime \prime \prime} & =\frac{1}{T}\left\{\frac{1}{T}\left[k\left(\frac{\partial T}{\partial x_{i}}\right)+\frac{4}{\kappa_{R}} \sum_{n=0}^{\infty}\left(\frac{\nabla^{2}}{\kappa_{M}^{2}}\right)^{n}\left(\frac{\partial E_{b} / \partial x_{i}}{2 n+3}\right)\right]\right. \\
& \left.\times\left(\frac{\partial T}{\partial x_{i}}\right)+2 \mu s_{i j} s_{i j}\right\}
\end{aligned}
$$

or, in terms of Eq. (14),

$$
\begin{aligned}
s^{\prime \prime \prime} & =\frac{1}{T}\left\{\frac { 1 } { T } \left[k\left(\frac{\partial T}{\partial x_{i}}\right)+\frac{1}{3 \kappa_{R}}\left(\frac{\partial J}{\partial x_{i}}\right)\right.\right. \\
& \left.+\frac{4}{3 \kappa_{R}} \sum_{n=0}^{\infty} \frac{4 n}{(2 n+1)(2 n+3)}\left(\frac{\nabla^{2}}{\kappa_{M}^{2}}\right)^{n}\left(\frac{\partial E_{b}}{\partial x_{i}}\right)\right] \\
& \left.\times\left(\frac{\partial T}{\partial x_{i}}\right)+2 \mu s_{i j} s_{i j}\right\}
\end{aligned}
$$

\section{Conclusions}

The radiative stress obtained from the specular (kinetic) moments of the transfer equation is considered. In terms of an isotropic radiative pressure, this stress is given an alternate form by following a development similar to that of the viscous (Stokesean) stress from the elastic (Hookean) stress.

An expression for local entropy production, including the effect of radiation as well as that of conduction and (viscous) friction, is developed. The radiative contribution to this production is expressed in terms of the radiative stress. Alternate forms of the entropy production are stated by considering the "elastic" and "viscous" equivalents of the radiative stress. These forms include also the usual contributions of conduction and (viscous) friction.

\section{References}

'Arpaci, V. S., "Hookean and Stokesean Implications of Radiative Stress," ASME Paper WA-85, 1985.

${ }^{2}$ Felske, J. D. and Tien, C. L., "The Use of the Milne-Eddington Absorption Coefficient for Radiative Heat Transfer in Combustion Systems," Transactions of ASME Journal of Heat Transfer, Vol. 99, 1977, pp. 458-465.

${ }^{3}$ Traugott, S. C., "Radiative Heat-Flux Potential for a Nongrey Gas," AIAA Journal, Vol. 4, 1966, pp. 541-542.

${ }^{4}$ Cogley, A. C., Vincenti, W. G., and Gilles, S. E., "Differential Approximation for Radiative Transfer in a Nongrey Gas near Equilibrium,"' AIAA Journal, Vol. 6, 1968, pp. 551-553.

${ }^{5}$ Lord, H. A. and Arpaci, V. S., "Effect of Nongray Thermal Radiation on Laminar Forced Convection over a Heated Horizontal Plate," International Journal of Heat and Mass Transfer, Vol. 13, 1970, pp. 1737-1750.

${ }^{6}$ Arpaci, V. S. and Gozum, D., "Thermal Stability of Radiating Fluids: The Benard Problem," The Physics of Fluids, Vol. 16, 1973, pp. 581-588.

${ }^{7}$ Arpaci, V. S, and Bayazitoglu, Y., "Thermal Stability of Radiating Fluids: Asymmetric Slot Problem," The Physics of Fluids, Vol. 16, 1973, pp. 589-593.

${ }^{8}$ Phillips, W. G. and Arpaci, V. S., "Monatomic Plasma-Thermal Radiation Interaction: A Weakly-Ionized Kinetic Model,' Journal of Plasma Physics, Vol. 13, 1975, pp. 523-537.

${ }^{9}$ Arpaci, V. S. and Tabaczynski, R. J., "Radiation-Affected Laminar Flame Propagation," Combustion and Flame, Vol. 46, 1982, pp. 315-322.

${ }^{10}$ Arpaci, V. S., “Radiation Affected Thermal Ignition," ASME Paper WA-84, 1984.

${ }^{11}$ Arpaci, V. S. and Tabaczynski, R. J., "Radiation-Affected Laminar Flame Quenching," Combustion and Flame, Vol. 57, 1984, pp. $169-178$.

${ }^{12}$ Unno, W. and Spiegel, E. A., "The Eddington Approximation in the Radiative Heat Equation,"'Publications of the Astronomical Society of Japan, Vol. 18, 1966, pp. 85-95.

${ }^{13}$ Milne, E. A., "Thermodynamics of Stars," Handbuch der Astrophysik, Vol. 3, 1930, Chap. 2, pp. 65-255.

${ }^{14}$ Arpaci, V. S. and Larsen, P. S., Convection Heat Transfer, Prentice-Hall, Englewood Cliffs, NJ, 1984, pp. 40-46.

${ }^{15}$ Sparrow, E. M. and Cess, R. D., Radiation Heat Transfer, Hemisphere Publishing Co., Washington, 1978, pp. 191-195.

${ }^{16}$ Ozisik, M. N., Radiative Transfer, Wiley-Interscience, New York, 1973, pp. 451-458.

${ }^{17}$ Siegel, R. and Howell, J. R., Thermal Radiation Heat Transfer, Hemisphere Publishing Co., Washington, DC, 1981, pp. 681-684.

${ }^{18}$ Vincenti, W. G. and Kruger, C. H., Introduction to Physical Gas Dynamics, John Wiley \& Sons, New York, 1965, pp. 473-476.

${ }^{19}$ Sampson, D. H., Radiative Contributions to Energy and Momentum Transport in a Gas, Wiley-Interscience, New York, 1965, pp. 28-37. 